\title{
Corrosion Cast- Comparison of Different Techniques and Materials Used in Corrosion Casting
}

\author{
Suma $\mathrm{H} \mathrm{Y}^{1}$ \\ ${ }^{1}$ Additional Professor, Department of Anatomy, Jawaharlal Institute of Postgraduate Medical Education and Research, Puducherry.
}

\section{Abstract}

In medical terms, cast refers to the replica obtained after filling a luminal structure with resin to reproduce its morphology. It can be accomplished by removing the soft tissue around it, termed corrosion or maceration. Corrosion casts form very important material in learning morphology of various structures like- vascular tree, tracheobronchial tree, luminal casts of various organs. Vascular casts have been studied using scanning electron microscopy, which has contributed to understanding the biological processes. If the specimen is fixed after resin injection, it can be used for surgical training, medical teaching etc. In the present review, the authors have tried to analyse the technical aspects of corrosion casting. We have also added note on polyurethane foam cast of coronary vessels, which was carried out in our laboratory.

Keywords: Corrosion cast, Silicone cast, Vascular casts, Polyurethane foam, Methyl methacrylate.

Corresponding Author: Dr. Suma H Y, Additional Professor, Department of Anatomy, Jawaharlal Institute of Postgraduate Medical Education and Research, Puducherry.

Received: February 2019

Accepted: February 2019

\section{Introduction}

Casting refers to filling of anatomical or pathological spaces with extraneous material which results in the production of a three-dimensional replica of the said space. ${ }^{[1]}$ In corrosion casting, the surrounding tissue of the casted hollow space is removed. The cast thus obtained represents the replica of the space of interest. Corrosion casts are useful in macroscopic as well as microscopic studies. Corrosion casts not only replicate the gross architecture but also the fine luminal surface morphology of the system cast. ${ }^{[2]}$

From the time, corrosion cast has been introduced different materials and corroding agents have been tried by scientists. The media include- gelatine, latex, silicone, Araldite, Plastoid, Methyl methacrylate, and recently Polyurethane foam (PU foam). Different casting media vary in terms of viscosity and polymerization times. The reagents used for maceration includes- acids or alkalis. Hence the protocols for corrosion casting varies in literature. Hence the present paper attempts to compare the various techniques adopted in different studies. We would also add a note about the technique adopted in our study using PU foam. This will be beneficial to conduct similar studies in the future.

The applications of corrosion cast are diverse. When used in macroscopic studies, they help to know the morphology in the normal and pathological specimen as well as variations. In the case of microscopic studies, Scanning Electron Microscope (SEM) has become an important tool in research. Studies were done using SEM focus on either change during embryological development, in interventional studies for vascular changes and to detect any changes in pathological states.

The corrosion cast can be done to study vascular, luminal morphology or cavities of organs. In the present paper, vascular casts will be discussed.

The steps in corrosion casting include: preparatory or precasting, casting proper, curing (post casting), corrosion, and last being post-processing.

\section{A. Pre-Treatment Of The Specimen Prior To Casting (Pre-Casting/ Preparatory Phase): \\ Type of specimen:}

Most studies consider fresh specimens to be always better for vascular corrosion. The biological specimen of interest is isolated and needs to be washed thoroughly. Once the organ is taken out, blood clot begins to form and the possibility of vasoconstriction due to neurogenic or metabolic stimuli exists. Blood or clot in vascular system can affect penetration of the casting material. ${ }^{[2,3]}$ The final outcome also depends on the age and condition of the specimen.

\section{Clearing of the lumen of the structure of interest:}

Heparin has been used in animal studies since it prevents clotting and flushing the blood vessels was easier. It has been added, prior to sacrificing the animal. Then the vessels were flushed with saline or ringer lactate (RL) under monitored pressure. ${ }^{[4]}$ Makanya et al (2007) suggested that vasculature needs to be irrigated with phosphate buffered saline (PBS), and can be mixed with anti-coagulant like 
heparin. ${ }^{[5]}$ But the effect of heparin on the outcome has not been described. ${ }^{[2]}$

Different studies mention the use of various solutions for washing and such solutions are alkaline in nature. Examples include phosphate buffered saline (PBS), Tyrode's solution, Ringer lactate (RL). They can be injected manually or using a perfusor. ${ }^{[6]}$ Prior treatment with formalin or any other method, leads to retraction and hardening of tissues. This may affect further steps in preparation. ${ }^{[3]}$

Whereas, Tompsett (1970) suggested the injection of tissues with $4 \%$ formalin prior to casting. They stopped perfusion at least 24 hours before resin injection, to facilitate the escape of excess fluid. ${ }^{[7]}$ Use of hydrogen peroxide $(1-2 \% \mathrm{H} 2 \mathrm{O} 2)$ has been suggested, to clean the lumen of clots or debris (Important to wash after $\mathrm{H} 2 \mathrm{O} 2$, otherwise $\mathrm{H} 2 \mathrm{O} 2$ itself may damage the tissue). ${ }^{[3]}$

\section{Vascular prefixation:}

Prefixation is an optional step, after the blood vessels are cleared. This helps in preventing resin extravasation into the tissues, vascular wall resistance is increased, reduces expansion of blood vessels while injecting the resin. The fixatives used include formaldehyde, glutaraldehyde, and paraformaldehyde in differing concentrations. This needs to be avoided when studying embryos, or lower concentration fixative can be used. ${ }^{[6]}$

Alvernia et al (2010) mention the need to irrigate arterial and venous system separately, arterial first (since venous congestion can increase arterial resistance and slow the flow). ${ }^{[8]}$ Mishra et al, in their study on placental vessels, flushed the vessels using saline, then water was removed with a dehydrating agent. ${ }^{[9]}$

Krucker et al (2006) perfused the blood vessels of mice and rat with $4 \%$ formalin in PBS for SEM study. ${ }^{[10]}$ Mazur M (2015) who studied fresh human hearts gave water wash and subsequent wash with prewarmed saline, ${ }^{[11]}$ Sordi N (2014), describe that the specimens which cannot be processed immediately can be refrigerated and injection can be done when convenient. They washed the vessels with tap water, then air dried them before the injection step. ${ }^{[12]}$ In our study, we also refrigerated a few hearts and washed them when convenient.

Aultman et al (2003) who worked with silicone and did luminal casts, placed the specimens in running water bath overnight. If they cannot be processed immediately, 3-5\% formalin was added. Water left in the lumen was washed with compressed air prior to injectioz. ${ }^{[13]}$ In case of Brain specimen, immersion in 5\% formalin for 3-5 days has been recommended so that it becomes firm and easier to handle. ${ }^{[8,13]}$

\section{Casting media:}

Prior to the study, the technique needs standardization in individual laboratories. Many casting media have been tried and used. The properties of ideal casting materials that have been described. ${ }^{[2,6]}$ It should be:

1) Non-toxic

2) Physiologically inert.

3) Polymerize within an adjustable period of time.

4) Should not shrink or lead to morphological changes in the tissues during polymerization.
5) Should not penetrate interstitial spaces of the tissues.

6) Should have a viscosity as to allow its flow through smaller blood vessels.

7) It should be corrosion resistant.

But the commercially available media do not meet all the criteria mentioned above.

Classification of casting materials: as per Kati Haenssgen, ${ }^{[1]}$

a) Aqueous solutions: India ink, Prussian blue

b) Flexible materials: gelatine, latex, silicone rubber

c) Hard materials: polyesters, epoxy resin, Methyl methacrylate (Mercox), polyurethanes.

Casting materials and their application in research and teaching have been exhaustively reviewed by Kati Haenssgen (2014). The cast material should be able to resist the critical steps like- during maceration, decalcification, and final cleaning. These processes involve immersion of cast in hot alkali $(\mathrm{NaOH}$ or $\mathrm{KOH})$ and or acids $(\mathrm{HCl}$, Trichloroacetic acid, formic acid or Tetrachloric acid). These reagents should not cause damage to the surface of the casts. ${ }^{[2]}$

The media used for casting vary in physicochemical properties- viscosity, replication quality, properties, corrosion resistance, thermostability. If the viscosity is high, the media cannot enter capillary bed or smaller arterioles and venules. Hence, they cannot be studied. The viscosity can be adjusted as per the requirements and instructions manual can be referred for the same purpose. ${ }^{[2]}$

The resins used for injection is said to consist of- polymer, plasticizer (softener), catalyst, accelerator (initiator). Resins are highly volatile, hence need to be handled with care. It has been advised to work under a fume hood with gloved hands. When handling ester compounds which are highly volatile- fume hood, ventilation at the workspace, gloves, protection for eyes are a must. ${ }^{[2]}$

\section{B. INJECTION TECHNIQUE PROPER: \\ Preparation of media:}

Some media like silicone and PU foam are available in canisters and can be used directly. Some need to be mixed with a solvent. After this, if any color is required, it can be added. The volume has to be determined prior so that the volume to be prepared is known.

Mixing time, injection duration and hardening time of the media.

The injection mixture needs to be injected, such that solidification doesn't start before the process is completed. If done too fast, can soak tissues and prevent cast from setting properly and making it fragile. Hence, the working life of the resin needs to be found out before the actual process. Working life has been defined as "the exact time the resin takes to solidify after the accelerator is added". ${ }^{[7]}$

To improve the clarity, colors can be added to the media before injecting. The established color codes for the structures are- red-artery, blue-vein, white- airway, yellowurinary ducts, green- biliary ducts system. ${ }^{[3]}$ Tompsett suggests avoidance of pigment pastes in corrosion casting, as they are resistant to acids. Pigment powders can be used instead. $^{[7]}$

Roberto JRE et al (2017) studied using acrylic, epoxy resin, polyester resin, and RTV Silicon and compared mixing time 
for the media, maximum injection time, hardening time. ${ }^{[3]}$ David Viggiano et al (2003) mention the hardening time for PU foam to be 2 hours. PU foam is resistant to $\mathrm{NaOH}$, hence used in corrosion. The foam when allowed to expand freely, it increases by $43 \%$ its volume. Whereas in a luminal structure, it is more compact. ${ }^{[14]}$

Method of injection: Most studies have mentioned the use of the syringe for injection, but some prefer using positive pressure since it can be controlled. The syringe volume depends on the quantity to be injected. For small specimen, $20 \mathrm{~mL}$; for large ones, $50 \mathrm{~mL}$ syringes will be convenient. Low viscosity resins are easy to inject. Some articles using silicone, mention use of Caulk gun.

Use of tube/cannula or syringe of appropriate size- Roberto JRE studied minute ducts and stressed the importance of using the tube of the correct size and it should permit injection of the polymer. They also suggested, if the proper diameter tube is not available, a smaller diameter tube is better. If bigger one is available, the terminal end can be cut in form of a wedge. ${ }^{[3]}$

\section{Perfusion pressure:}

The process of perfusion helps to flush the circulatory system and removes any obstacles. For macroscopic corrosion casting simple syringe suffices for injection. But for larger organs, or for SEM studies, injection at constant pressure is essential. Hence, two types of perfusion apparatus have been described- irrigators and perfusers. Irrigators are gravity based, which are fixed at a height to allow the flow of resin. But perfusers are motor-driven, which can hold syringes of different sizes and able to produce controllable flow rate. ${ }^{[2]}$

Hossler and Douglas (2001), who worked on blood vessels of small animals, mention the importance of monitoring the injection pressure. It varies as per the animal or the specimen. Hamster heart was found to require $80 \mathrm{mmHg}$ of pressure, whereas for hamster lung it was $15 \mathrm{mmHg}$.

Verli, et al (2007), opined that the pressure at which injection and infusion are done to be properly tuned, to avoid incomplete filling or rupture of structures and homogenous cast can be obtained. Manual injection is commonly used. But if injection apparatus is used, constant pressure can be maintained and prevents air bubble. ${ }^{[6]}$

\section{How to avoid spatial distortion:}

Tompsett devised a standard apparatus for resin injection. The pressure was applied onto the glass tube with help of enema syringe. The specimen was placed in a water bath, which preserves the anatomy. ${ }^{[7]}$

The specimen can get distorted during the various steps of casting as discussed by Aharinejad. They include- a) positioning of the specimen can compress along one surface, b) high injection pressure, c) too strong solutions used during corrosion step or for a longer duration, d) during air drying by high surface tension when water evaporates. ${ }^{[2]}$

\section{Suture material:}

Appropriate materials for clamping or suturing needs to be kept ready before the injection. Some blood vessels which can lead to leakage needs to be tied beforehand. In the case of larger vessels, silk thread is recommended as it is easy to handle. If the diameter of the lumen is same as the external diameter of the cannula, a simple ligature is sufficient. But for smaller vessels, monofilament suture material has been suggested. ${ }^{[2]}$

It is important to secure the cannula within the lumen of the vessel/duct or any other tubular structure using appropriate suture material. Due to injection pressure, there exists every possibility of extravasation of the resin.

\section{Injection step:}

Following end points have been mentioned across studies so that further injection of media can be stopped. ${ }^{[1]}$

a) Once the media start appearing at the venous end.

b) Casting material shines through skin.

c) Resistance is felt while injecting.

Following is the list of tips suggested in articles, which can be helpful while casting:

a) While injection is about to get over, the media from syringe should be pushed fully, as air bubbles can be introduced.

b) When the tissue is placed on a solid surface, tissue damage can occur. Some studies have suggested using tissue floatation baths to avoid this.

c) When multiple vessels need to be injected simultaneously, a four-way system of injection is recommended.

d) In the case of partially blocked luminal structures, the use of negative pressure could be helpful. ${ }^{[1]}$

\section{POST-CASTING:}

It should be left untouched for at least half an hour. Some authors allow polymerization to be carried out in a water bath to avoid spatial distortion. Verli et al, describe immersion of cast in warm water at $30-600 \mathrm{C}$ for a duration of 30 minutes to 24 hours to allow polymerization of the resin. ${ }^{[6]}$ Some have used refrigeration so that the cast can set. ${ }^{[12]}$ Krucker et al. (2006) placed the specimen in vacuum for 2-3 $\mathrm{min}$ to remove air inclusions in the resin. ${ }^{[10]}$ For some media, the specimen needs are to be placed in an incubator to allow polymerization. ${ }^{[9]}$ In our study, the specimen was refrigerated overnight at $40 \mathrm{C}$.

\section{CORROSION:}

Corrosion step is used to remove soft tissue around the cast. It can be carried out by biological or chemical methods as discussed by Kati Haenssgen. ${ }^{[1]}$

a) Biological Corrosion Casting:

* In unfixed specimens, tissue destruction occurs by the action of endogenous (Autolysis) and microbial enzymes (Putrefaction). But it is not suitable.

* Carrion insects.

* Enzymatic maceration.

* The decay of organic material- by larvae and maggots, clears the soft tissue. Some use them for delicate structures.

* Boiling in water.

\section{E. Chemical methods:}

* Ariel (detergent).

* Enzymes- pancreatin, papain.

* Strong alkalis- $\mathrm{KOH}$ (potassium hydroxide) 
* Acids- $\mathrm{HCl}$ (Hydrochloric acid), H2SO4 (Sulphuric acid).

In the following section, highlights of different studies in relation to the injection technique part have been discussed: Hossler and Douglas (2001) did SEM study on blood vessels of animals using Mercox resin. Maceration was carried out with $20 \% \mathrm{NaOH}$ or $5-20 \% \mathrm{KOH}$ alternated with hot water rinses. The casts were cleaned using formic acid and distilled water, air dried and lyophilized before further study. ${ }^{[4]}$

$\mathrm{Mu}$ et al (2001) worked with latex on placental vessels and cotyledons. The collected placentas were placed in a solution of saline and heparin. They allowed for spontaneous drainage of blood. Then they flushed the placental vessels till the effluent was clear. Then they were immersed in a $12.5 \%$ glutaraldehyde solution for fixation. Finally, red latex was injected and left for polymerization. Maceration was carried out with $35 \% \mathrm{HCl}$, for 3 to 4 weeks. They didn't mention regarding the need to change the solution frequently. ${ }^{[15]}$

Aultman et al (2003) did study on tracheobronchial tree, cardiac vessels and brain ventricles. They used RTV Silicone to which curing agent and coloring agent were mixed. The injection apparatus consisted of vinyl tubing with screw type syringe adapter fitted to one end. The oozing of media was prevented by clamps or waxed strings. Then it was allowed to harden at room temperature for 24-48 hours. The corrosion was carried out with freshly prepared $10 \% \mathrm{KOH}$. This required 5-7 days following which they were placed in boiling water for 8-12 hours to clear the tissues from the polymer. Then specimens were placed in $5 \%$ hydrogen peroxide for about 2 hours for removal of residual tissues. Casts were then rinsed in running water overnight. ${ }^{[13]}$

David Viggiano et al (2005) studied Broncho vascular tree and vascular tree of kidney of animals by PU foam. They used compressed air device with the PU foam resin, which was held upside down for uniform injection pressure. Injection was stopped when the resin starts appearing on small vessels on the surface of the organ. The cannula was left for 20 minutes without disturbance, to allow polymerization. Corrosion was done using $10 \% \mathrm{NaOH}$, and the liquid was changed every day. Then it was washed and air dried. ${ }^{[14]}$

Krucker et al (2006) worked using PU4ii (Polyurethane), Mercox (Acrylic based), and Clear Flex (Urethane based) on mouse brain blood vessels. Polyurethane-based resin (PU4ii) was found ideal for SEM since dense capillary beds fill well. They used Ethyl methyl ketone (EMK) or dichloromethane (DCM) as diluent at a concentration of $40 \%$. The volume of the resin was five times the physiological blood volume. To the resin/solvent mixture, hardener was added with a pipette (6:1 ratio) on a vortex mixer, then transferred to a syringe. The mixing was carried out in glass or plastic vial. The prepared resin mix was placed in vacuum for few minutes, to remove air inclusions. The curing was carried out at room temperature for 2 days (for polymerization). Tissue maceration was carried out with $7.5 \% \mathrm{KOH}$ for 24 hours at $500 \mathrm{C}$, then washed with water and freeze-dried.

Verli et al (2007), mention the use of detergent along with $\mathrm{NaOH}$ and found that it enhances the process of maceration.
They also mention the use of other chemicals which could be used prior to drying like- a) 5\% Formic acid for 5-30 min, or b) Collagenase with HCL or c) Increasing concentrations of alcohol. ${ }^{[6]}$

Nadia Sordi (2014) studied vasculature in animals using PU foam. The PU foam media was sprayed into a container and mixed with acetone that prevents its expansion. Nitro dyes were added at this stage and mixed well. Then, as per the volume of foam, a syringe was selected and media filled in it for injection. The authors described the ratio of foam: acetone and the time required to perform the injection in their article. The specimen was suspended in anatomical position and stored overnight at $40 \mathrm{C}$. Finally, corrosion was done using either $30 \% \mathrm{H} 2 \mathrm{SO} 4$ solution or in a $10 \% \mathrm{NaOH}$ solution. Large specimen required fluid change every 5-7 days. They suggested use of foam cupboard for storing the sample. After 24-48 hours, it was washed with jets of water and then air dried. ${ }^{[12]}$

Patel J et al (2014): the prepared specimen (placental vessels) with Cellulose acetate butyrate granules (CABG). $\mathrm{CABG}$ was mixed with acetone in the ratio of $1: 2$. The injected specimens were transferred to a mixture of formalin and saline for 48 hours. Corrosion was carried out with a mild solution of $\mathrm{KOH}(5 \%)$ in a glass jar for the duration of 24 hours. They traced the blood vessels of placenta under a constant stream of running water which washed remaining parenchyma. ${ }^{[16]}$

Mazur M (2015): (worked with Duracryl Plus on coronary venous system) after the cast is set, the specimen was washed with warm distilled water, several times. Corrosion was carried out with $10-15 \%$ solution of $\mathrm{KOH}$ which required three weeks in case of heart. The solution was replaced every day after a wash with distilled water. Tissue fragments still remaining were cleaned with $3 \%$ Trichloric acid solution. Then they were rinsed again in distilled water. $^{[11]}$

Mishra et al (2015)- They worked with Polyvinyl chloride (PVC) on the placental vessels. PVC is a thermoplastic polymer with $57 \%$ chlorine and $43 \%$ carbon used in the plastic industry. It is available as a colorless viscous liquid and diluted in a ratio of $1: 1$. The colored resin was added and filled in the syringe. The injection was continued till resistance was felt, and mention that the process needs to be completed within one hour to prevent early polymerization. After the cast is set (overnight incubation at $800 \mathrm{C}$ ), the specimen was washed with tap water. Then immersed in a large glass container containing 50\% hydrochloric acid $(\mathrm{HCl})$, which corroded the parenchyma. Subsequently, it was washed and dissected clean with forceps. ${ }^{[9]}$

They found that PU foam was superior compared to other materials, in terms of replication, elasticity and durability, doesn't produce unnecessary extravasation. It is also easy to store, convenient to use with larger specimens. It also has drawbacks which include- toxicity, short shelf life after the storage container is opened, need for good ventilation and other universal precautions. ${ }^{[10]}$

Roberto JRE et al (2017)- used Acrylic as the media. Other media used in the study were- epoxy resin, polyester resin, RTV silicone, and polyurethane foam. The study mentions the concentrations, uses, mixing and injection time for each, and characteristics of different injectable materials. The 
study highlights method of dissecting ducts of the organs to be studied, and different type of sutures and suture materials so that cannula can be secured in place. They utilized positive pressure compressor for injection. When done manually, syringes of different sizes were used depending upon the specimen. Once injection was completed, clamps were placed to prevent reflux, and allow for polymerization. Then the specimen was placed in anatomical position or specimen was kept submerged in sodium chloride solution or water. They suggested that time required for polymerization could be altered by a catalyst. The corroding agent used was $\mathrm{NaOH}$. The precautions to be taken while working with $\mathrm{NaOH}$ include- steel containers to be used (iron or aluminium containers corrode with $\mathrm{NaOH}$ ), the need for neutralizing it with weak acid (like vinegar) before disposing. ${ }^{[3]}$

In our study, we used glass beakers of appropriate capacity for mixing the media with solvent. (PU foam with acetone plus color). The PU foam (in grams) and acetone (in $\mathrm{mL}$ ) was mixed in the ratio of 4:1. For one heart, 15-20 grams of PU foam is required for filling the coronary venous tree. It has to be done just before injection, otherwise, polymerization begins. We used a modified endotracheal tube with a syringe attached for cannulating the coronary sinus. The inflatable bulb of the ET tube prevents backflow of the resin.

After casting, the specimen was placed in a refrigerator at 240C overnight. Then it was taken out and left for thawing. Corrosion was carried out with $10 \% \mathrm{NaOH}$ (1.5-1.8 L was required for a heart specimen) for 3-5 days. The solution required a change after 48 hours. After maceration is complete, the specimen is washed with water. But due to saponification, cast. This was overcome by immersing the cast in $800-1000 \mathrm{~mL}$ of Triton-X 100 in an incubator at 45500C for 24-48 hours. Then final washing is done and air dried.

\section{F. POST PROCESSING:}

In relation to post-processing, not many articles mention difficulties encountered once the cast is ready, and the methods to overcome it.

Tompsett has described techniques to handle the casts after they are prepared. The casts tend to break if brittle, hence spectacles should be always worn while working at this step since flying fragments can be harmful. Any broken fragments should be preserved, as they can be useful in repairs of the cast. Resin cement has been recommended for sticking fragments. They also suggest the use of a spray of Marco by which fragile cast is strengthened. For further details, refer to Tompsett- Anatomical techniques. ${ }^{[7]}$

In the present study, if any fragment was broken, was fixed using epoxy-based resin.

\section{Conclusion}

The technique of corrosion cast provides excellent opportunity to understand the vascular pattern as well as morphology of organs with lumen. There are various resins available in the market which can be chosen as per the structure of interest and laboratory conditions. In institutes where facilities are available, scanning electron microscopy of vascular structures can be carried out.

In the present article, we have tried to present overview of the steps and difficulties encountered during corrosion casting. It is beneficial for researchers as well as academicians. We did casting with polyurethane foam on coronary blood vessels. The advantages of PU foam are- the ease of preparation, it is economical, can be carried in small laboratories and production of casts with finer details. The disadvantages include- necessity of fresh specimen and the casts are little brittle, hence require careful handling.

\section{References}

1. Haenssgen K, Makanya AN, Djonov V. Casting materials and their application in research and teaching. Microsc Microanal. 2014; 20:493-513

2. Aharinejad SH, Lametschwandtner A. Microvascular corrosion casting in scanning electron microscopy: techniques and applications. Springer Science \& Business Media; 2012 Dec 6.

3. Roberto JRE, Juan SLM, Daniel RMP, Juan DHR. Corrosion casting, a known technique for the study and teaching of vascular and duct Structure in Anatomy. Int. J. Morphol. 2017;35(3):11471153.

4. Hossler FE, Douglas JE: Vascular corrosion casting: review of advantages and limitations in the application of some simple quantitative methods. Microsc Microanal. 2001; 7: 253-64.

5. Makanya A, Gachoka J, Kariuki GK. A short review of the methods used in museum technology. Bull Anim Health Prod Afr. 2008; 56: 267-9.

6. Verli FD, Rossi-Schneider TR, Schneider FL, Yurgel LS, de Souza MA. Vascular corrosion casting technique steps. Scanning. 2007; 29:128-32.

7. Tompsett DH. Anatomical techniques. Edinburgh \& London, Churchill Livingstone; 1970.

8. Alvernia JE, Pradilla G, Mertens P, Lanzino G, Tamargo RJ. Latex injection of cadaver heads: Technical note. Neurosurgery, 2010; 67:362 -67.

9. Mishra S, Sethi M. A Reformed Corrosion Cast Technique using Commercially Available Polyvinyl Chloride Solution. MAMC J Med Sci. 2015; 1:28-30.

10. Krucker T, Lang A, Meyer Ep. New polyurethane-based material for vascular corrosion casting with improved physical and imaging characteristics. Microsc Res Tech. 2006; 69:138-147.

11. Mazur M, Walocha K, Kuniewicz M, Wandzel-Loch B, Tomaszewska I, Konarska M et al. Application of Duracryl plus for preparation of corrosion casts of venous coronary tree of human heart. Folia Medica Cracoviensia. 2015; 55(3): 69-75.

12. De Sordi N, Bombardi C, Chiocchetti R, Clavenzani $P$, Trerè $C$, Canova $\mathrm{M}$ et al. A new method of producing casts for anatomical studies. Anat Sci Int. 2014; 89:255-65.

13. Aultman A, Blythe AJ, Sowder H, Trotter R, Raoof A. Enhancing the value of organ silicone casts in human gross anatomy education. J Int Soc Plastination. 2003; 18:9-13.

14. Viggiano D, Sangiorgi S, Requzzoni M, Marano MR, Dell ${ }^{\text {‘eOrbo }}$ $\mathrm{C}$ et al. A new method to make vascular and bronchial casts of voluminous organs. Eur J Morphol. 2003; 41:161-5.

15. J M, Toru K, Takuji T, Hirotsugu F, Eriko F, Shinji F, Kenshi Wa et al. A comparative study of intraplacental villous arteries by latex cast model in vitro and color Doppler flow imaging in vivo. J. Obstet. Gynaecol. Res. 2001;7(5): 297-303.

16. Patel J, Patel B, Dave R, Samir Ram, Bhojak N, Desai J. A study of placental vascular pattern by corrosive cast in Gujarat region. NJIRM. 2014; 5(1): 64-71. 


\section{Suma; Different Jechniques and Materials Used in Carrasian Casting}

Copyright: (C) the author(s), publisher. Academia Anatomica International is an Official Publication of "Society for Health Care \& Research Development". It is an open-access article distributed under the terms of the Creative Commons Attribution Non-Commercial License, which permits unrestricted non-commercial use, distribution, and reproduction in any medium, provided the original work is properly cited.

How to cite this article: Suma HY. Corrosion Cast- Comparison of Different Techniques and Materials Used in Corrosion Casting. Acad. Anat. Int. 2019;5(1):25-30.

DOI: dx.doi.org/10.21276/aanat.2019.5.1.7

Source of Support: Nil, Conflict of Interest: None declared. 